\title{
Portable Power Bank, Dare to Ask Where the Road is -- Survey of the Capital University Student Charging Treasure
}

\author{
Minghao Chen, Yang Yang and Yuwen Hui \\ North China Electric Power University, Beijing, 102206 \\ alanncepu@foxmail.com
}

Keywords: Questionnaire survey; Rechargeable treasure; Feasibility; Drawbacks

\begin{abstract}
Based on the data collected from the questionnaire survey, this paper analyzes the current situation of the use of rechargeable treasure of the university students in the capital, and then obtains the characteristics of the usage of the charging treasure of the university students. Then, the paper analyzes the main problems existing in the traditional charging treasure, finally probes into the new mode of the shared charging treasure which changed "buys" as "rents", and the feasibility and concrete measures to introduce and develop in the university, and analyzes the drawbacks of its existence.
\end{abstract}

\section{Status and Analysis}

With the increasing reliance on mobile phones, the attendant problem of portable charging is also troubling more and more people. According to the Ministry of Statistics shows that by the end of March 2017, the total number of mobile phone users in China reached 1.345 billion, but $78 \%$ of the cell phone battery capacity is distributed between 2000 Mah 4000mah, the demand for electricity and battery performance is a huge gap. The rapid consumption of electricity has led to the increase of the number of charges, making the charging treasure a strong just need. As a result, rechargeable treasure is playing a more and more important role in solving the portable charging tool.

In the one-year survey, we visited 13 universities in Beijing, and through the 1734 effective questionnaires collected from them, we got the relevant data of the use of rechargeable treasure by university students in the capital. Then, we have a systematic summary of the characteristics of the rechargeable treasure market, and get the following conclusions:

1. High prevalence, nearly $90 \%$ college students have rechargeable treasure.

According to our questionnaire, $87 \%$ of college students use rechargeable treasure in their daily life, which shows that the use of rechargeable treasure is universal among college students, and also reflects the demand of portable charging market from the side. In addition, our data also shows that $85 \%$ of college students in daily life will be frequent or frequent use of rechargeable treasure, which in large part for the future development of rechargeable treasure laid the foundation.

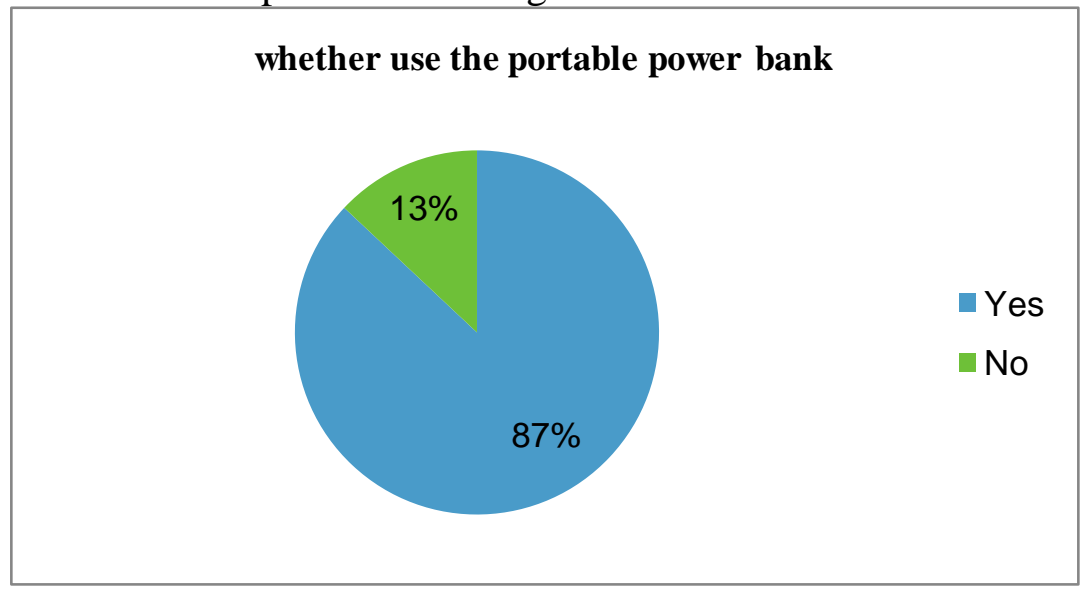

Figure 1. Whether use the portable power bank

2. The capacity is small; the charge treasure capacity is medium partial small mainly. 
According to our survey, 5000 Mah 1000 's medium-capacity rechargeable treasure is more acceptable to the student community in the rechargeable treasure already owned by college students, and the medium-small capacity has a better market prospect in the charging treasure that college students tend to have. Usually, small capacity means light weight, indicating that college students in the use of rechargeable treasure process, the same emphasis on the lightweight and portable charge.

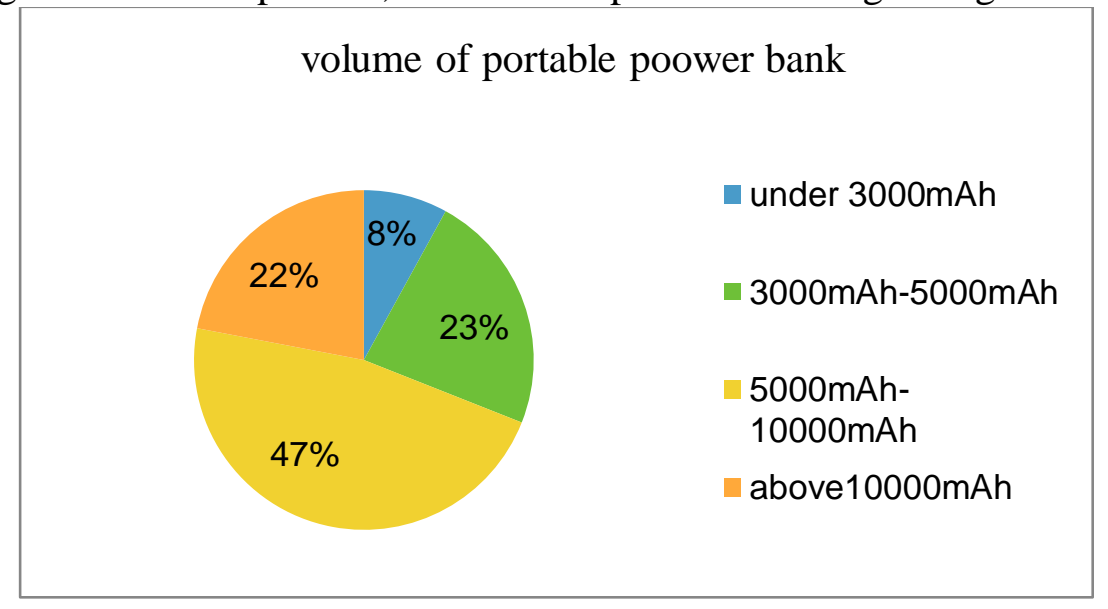

Figure 2. volume of portable power bank

3. Preference for low prices, college students are more sensitive to charge Bao price.

According to our findings, $73 \%$ of college students are ideally priced at between 75 and 125 yuan for charging treasure. We think that college students are more sensitive to price than the average working class, so they will be more cautious when purchasing and charging, in order to pursue higher cost-effective.

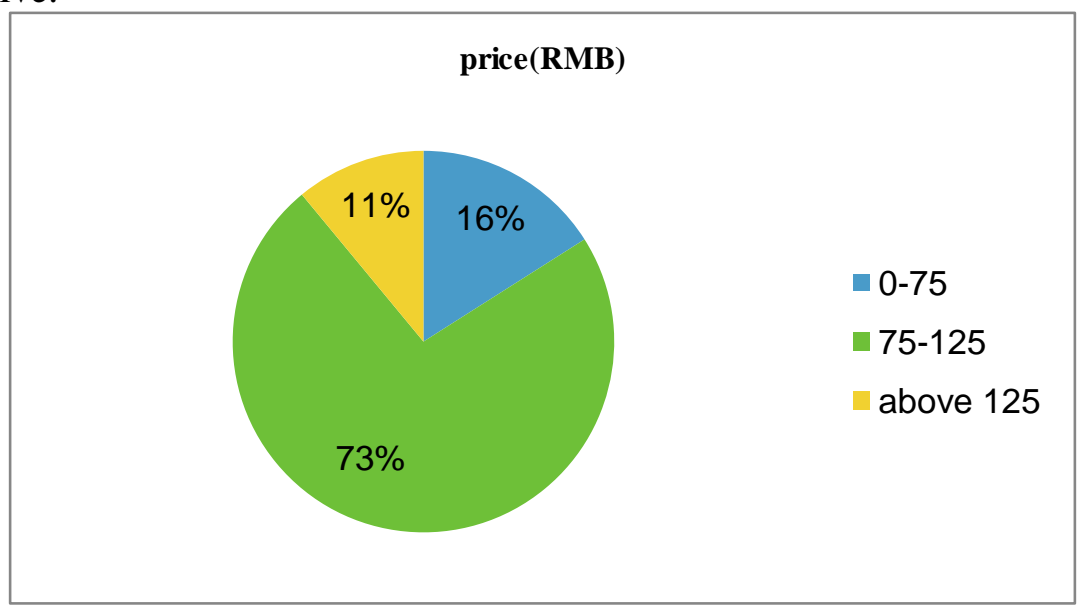

Figure 3. The price of the rechargeable treasure

4. Brand convergence, college students do not pursue brand effect.

According to our survey, Millet has the largest market share (about 29\%) among the many brands of rechargeable treasure, but the data also shows that the differences between the brands are not obvious. College students in the charging treasure to buy, more value is the size of the capacity, security and price, brand is not its focus. This also reflects from the side: The charge of the brand has a certain degree of convergence; the individual big brands also do not have a very prominent competitive point. 


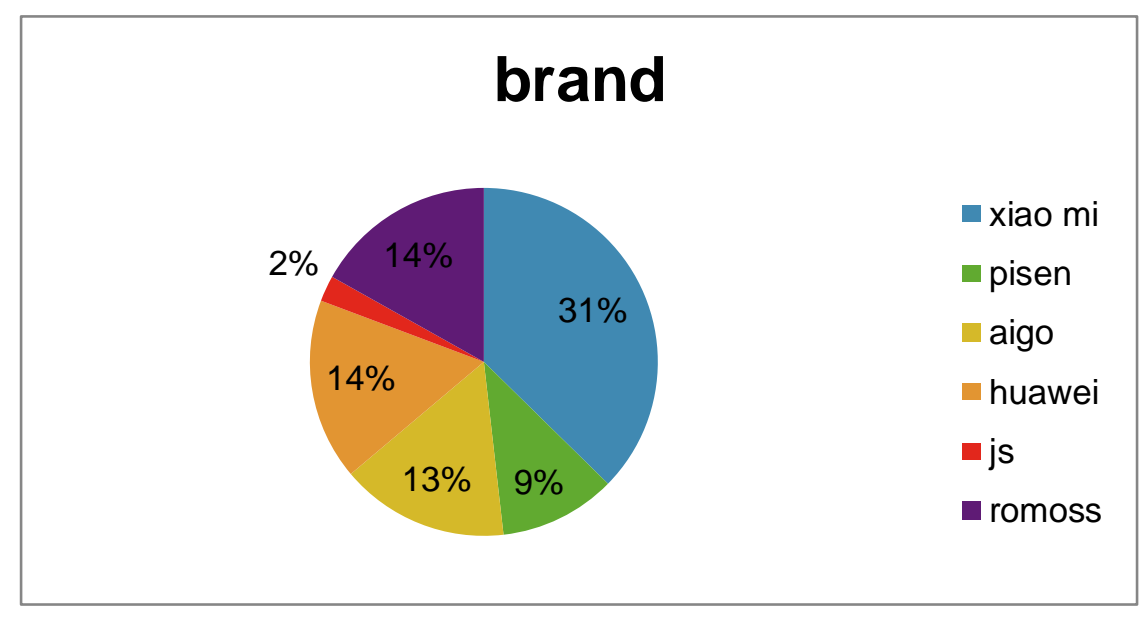

Figure 4. The brand of the rechargeable treasure

\section{Main Issues}

In this chapter, we deeply discuss the characteristics given above in detail, and combine the feedback which we received in the questionnaire to survey and analyze them. Finally, we find out the following problems in the model of traditional portable power bank.

1. Large quality reduce its portability. Due to the difficulty of meeting the demand between the large capacity and small quality of the portable power bank, the large capacity will inevitably lead to higher quality, which leads to the portability of the portable power bank will be numerously reduced. There is a new cyber word, "red panic",means people is flustered when their cellphone's battery color is red. This is a common occurrence among college students. For college students with strong cell phone dependence, it is undoubtedly a big problem to the desire to play cellphones and suffer the inability to charge. Therefore, the contradiction between the large capacity and the small quality of the portable power bank is the bottleneck of the model of traditional portable power bank.

2. Deficiency of market purchasing power. Only 31 percent of college students have the desire to purchase a new portable power bank to replace the old one, which makes the purchase potential of the portable power bank market inadequate. On the one hand, the inherent malpractice of the portable power bank in the quality has led to the generally poor usage experience for college students in the use of portable power bank. On the other hand, the university student is more sensitive to price, which indirectly leads to the low proportion of the number of students who have plan to replace the portable power bank. The purchase potential of the portable power bank market is insufficient, and the using mode of traditional portable power bank is still in the transition space and necessary.

3.Frequency of using is booming. According to the questionnaire, $85 \%$ of college students use the portable power bank more frequently, and the college students who rarely use the portable power bank are only a small minority. Due to relatively dependent on college students of electronic products, battery charging demand of the rapid consumption increasing, charging times also grow, so the using frequency of the portable power bank into a rising trend.

Therefore, portable power bank is the daily necessities of college students. College students not only hope to enjoy the immediate convenience of the portable power bank, but also wants to avoid its massive quality, however, they does not have the desire to buy substitute. Therefore, introducing a new kind of portable power bank usage pattern is imperative.

\section{Countermeasures and Analysis}

Nowadays, the Shared economy has become a big hit, from sharing bikes to sharing cars, sharing umbrellas and sharing homes. Sharing economy almost everywhere. We believe that the future can try to charge the usage pattern of portable power bank from "buy" to "rent" to give full play to the 
advantages of Shared economy in college students groups, exploring and developing a new mode which combining sharing economy and portable power bank.

Sharing portable power bank is similar to Shared cycling, which refers to pay the rent and scan the QR code from the facility of portable power bank to acquire a portable power bank. The facility in the process of charging on time or pay-per-view new charging mode. It has retained the advantage of the portable power bank, and has solved the disadvantages of the inconvenience caused by the high capacity and quality of the traditional portable power bank. In addition, with the cut off electric power of university dormitory at night time, Shared portable power bank effectively solves the inconvenience of traditional portable power bank at night, so the share portable power bank in college students groups have a good prospects for development. During the survey, we also pay close attention to the development of shared charge. At present, there are three kinds of shared charging modes in the society: one is to "call" as the representative of the mobile model of mobile sharing, the use of a single dozens of rechargeable treasure of large-scale machine services shopping malls, stations, hospitals and other places of mass flow; second, "Street power" as the representative of the fixed scene of mobile sharing, the use of a number of small-scale terminal machine in cafes, restaurants, counters and other small scenes; three is "small electricity" for the representative of fixed-scene fixed sharing, using the desktop charging pile or portable power bank. The same applies to restaurants and other small scene locations.

By comparing university environment and social environment, we find that the university environment has strong crowd mobility, the flow is large and the population flow has a strong timeliness and regional characteristics, that is, in the morning and afternoon period, the teaching area will be relatively concentrated, and in the meal time, the crowd will be concentrated in the canteen area; So the relatively concentrated environment of this group is conducive to the development of the university in the "call" represented by a large number of charging treasure of the terminal sharing mode.

In addition, we visited a number of shopping malls, stations, hospitals in a variety of shared charging terminal machine, found that the current social charging treasure rental model defects, that is, the use of fewer people. In this regard, we think there are two reasonable explanations. First, people to the new shared charging mode also to watch its change of attitude, so can not timely enjoy the convenience of sharing patterns. Second, the new charging mode still faces multiple security risks, in which information disclosure is one of the biggest concerns. These are the future sharing of the charging mode to put forward higher requirements.

\section{Conclusion}

To sum up, the traditional mode of portable power bank is widely accepted, so the market share is large, with a great advantage, but with the gradual improvement of the sharing economy, the drawbacks of traditional portable power bank also revealed. Among them, due to the large quality caused by the inconvenience of carrying, seriously restricting the traditional charging treasure further development, in order to solve this shortcoming, the sharing portable power bank is gradually accepted by people. The sharing of rechargeable treasure with its "at any time" "everywhere" advantage, quickly captured the masses, especially college students Heart, so that the sharing of rechargeable treasure in the campus development has a better prospect.

Admittedly, the portable power bank also has its own flaw, how to guarantee the user's information security bears the brunt. In the rapid development of information today, the sharing of charging mode to develop, the most important is the security problem, only to solve this problem, can be more people's favor.

\section{Reference documents}

[1] Ministry of Industry. Ministry of Industry issued 2017 1-March communications Business Economic performance [J]. Mobile communications, 2017 ,41(8):96-96.

[2] http://tech.sina.com.cn/mobile/n/c/2016-06-30/doc-ifxtsatm1036852.shtml. 
[3] Anon. Shared charge Treasure [J]. Shanghai Informatization, 2017 (5): 8-9.

[4] Anon. The profit pattern is not clear--sharing charge treasure solves is real pain point or pseudo demand. PC Digest Magazine, 2017 (9):12-13.

[5] Yao Youming, Meng Hanqi. Who makes the portable power bank so dangerous. Legality and economy Tri-Monthly, 2013 (12).

[6] Jin, Liuyang et al. Research on innovative design and application of wireless portable power bank. Private Technology, 2016 (11): 29-29.

[7] Anon. Who stole the power of the portable power bank. Intelligent mathematics, 2015 (12):1213.

[8] Anon, Borrow a portable power bank, mobile phone privacy was stolen. Mass standardization, 2015 (1):45-45.

[9] Zhang, Jisheng et al. Design of portable power bank battery management. Auto Time, 2017(4):71-75.

[10] Guo, Chaofei. Two suspense of portable power bank. China Entrepreneur, 2017 (10). 